\title{
APPENDIX
}

The least primitive root $g$ modulo $p$ for each prime $p<4000$ is given in the following table.

\begin{tabular}{|c|c|c|c|c|c|c|c|c|c|c|c|c|c|}
\hline$p$ & $g$ & $p$ & $g$ & $p$ & $g$ & $p$ & $g$ & $p$ & $g$ & $p$ & $g$ & $p$ & $g$ \\
\hline 2 & 1 & 101 & 2 & 233 & 3 & 383 & 5 & 547 & 2 & 701 & 2 & 877 & 2 \\
\hline 3 & 2 & 03 & 5 & 39 & 7 & 89 & 2 & 57 & 2 & 09 & 2 & 81 & 3 \\
\hline 5 & 2 & 07 & 2 & 41 & 7 & 97 & 5 & 63 & 2 & 19 & 11 & 83 & 3 \\
\hline 7 & 3 & 09 & 6 & 51 & 6 & 401 & 3 & 69 & 3 & 27 & 5 & 87 & 5 \\
\hline 11 & 2 & 13 & 3 & 57 & 3 & 09 & 21 & 71 & 3 & 33 & 6 & 907 & 2 \\
\hline 13 & 2 & 27 & 3 & 63 & 5 & 19 & 2 & 77 & 5 & 39 & 3 & 11 & 17 \\
\hline 17 & 3 & 31 & 2 & 69 & 2 & 21 & 2 & 87 & 2 & 43 & 5 & 19 & 7 \\
\hline 19 & 2 & 37 & 3 & 71 & 6 & 31 & 7 & 93 & 3 & 51 & 3 & 29 & 3 \\
\hline 23 & 5 & 39 & 2 & 77 & 5 & 33 & 5 & 99 & 7 & 57 & 2 & 37 & 5 \\
\hline 29 & 2 & 49 & 2 & 81 & 3 & 39 & 15 & 601 & 7 & 61 & 6 & 41 & 2 \\
\hline 31 & 3 & 51 & 6 & 83 & 3 & 43 & 2 & 07 & 3 & 69 & 11 & 47 & 2 \\
\hline 37 & 2 & 57 & 5 & 93 & 2 & 49 & 3 & 13 & 2 & 73 & 2 & 53 & 3 \\
\hline 41 & 6 & 63 & 2 & 307 & 5 & 57 & 13 & 17 & 3 & 87 & 2 & 67 & 5 \\
\hline 43 & 3 & 67 & 5 & 11 & 17 & 61 & 2 & 19 & 2 & 97 & 2 & 71 & 6 \\
\hline 47 & 5 & 73 & 2 & 13 & 10 & 63 & 3 & 31 & 3 & 809 & 3 & 77 & 3 \\
\hline 53 & 2 & 79 & 2 & 17 & 2 & 67 & 2 & 41 & 3 & 11 & 3 & 83 & 5 \\
\hline 59 & 2 & 81 & 2 & 31 & 3 & 79 & 13 & 43 & 11 & 21 & 2 & 91 & 6 \\
\hline 61 & 2 & 91 & 19 & 37 & 10 & 87 & 3 & 47 & 5 & 23 & 3 & 97 & 7 \\
\hline 67 & 2 & 93 & 5 & 47 & 2 & 91 & 2 & 53 & 2 & 27 & 2 & 1009 & 11 \\
\hline 71 & 7 & 97 & 2 & 49 & 2 & 99 & 7 & 59 & 2 & 29 & 2 & 13 & 3 \\
\hline 73 & 5 & 99 & 3 & 53 & 3 & 503 & 5 & 61 & 2 & 39 & 11 & 19 & 2 \\
\hline 79 & 3 & 211 & 2 & 59 & 7 & 09 & 2 & 73 & 5 & 53 & 2 & 21 & 10 \\
\hline 83 & 2 & 23 & 3 & 67 & 6 & 21 & 3 & 77 & 2 & 57 & 3 & 31 & 14 \\
\hline 89 & 3 & 27 & 2 & 73 & 2 & 23 & 2 & 83 & 5 & 59 & 2 & 33 & 5 \\
\hline 97 & 5 & 29 & 6 & 79 & 2 & 41 & 2 & 91 & 3 & 63 & 5 & 39 & 3 \\
\hline
\end{tabular}




\begin{tabular}{|c|c|c|c|c|c|c|c|c|c|c|c|c|c|}
\hline$p$ & $g$ & $p$ & $g$ & $p$ & $g$ & $p$ & $g$ & $p$ & $g$ & $p$ & $g$ & $p$ & $g$ \\
\hline 1049 & 3 & 1229 & 2 & 1429 & 6 & 1597 & 11 & 1783 & 10 & 1993 & 5 & 2161 & 23 \\
\hline 51 & 7 & 31 & 3 & 33 & 3 & 1601 & 3 & 87 & 2 & 97 & 2 & 79 & 7 \\
\hline 61 & 2 & 37 & 2 & 39 & 7 & 07 & 5 & 89 & 6 & 99 & 3 & 2203 & 5 \\
\hline 63 & 3 & 49 & 7 & 47 & 3 & 09 & 7 & 1801 & 11 & 2003 & 5 & 07 & 5 \\
\hline 69 & 6 & 59 & 2 & 51 & 2 & 13 & 3 & 11 & 6 & 11 & 3 & 13 & 2 \\
\hline 87 & 3 & 77 & 2 & 53 & 2 & 19 & 2 & 23 & 5 & 17 & 5 & 21 & 2 \\
\hline 91 & 2 & 79 & 3 & 59 & 5 & 21 & 2 & 31 & 3 & 27 & 2 & 37 & 2 \\
\hline 93 & 5 & 83 & 2 & 71 & 6 & 27 & 3 & 47 & 5 & 29 & 2 & 39 & 3 \\
\hline 97 & 3 & 89 & 6 & 81 & 3 & 37 & 2 & 61 & 2 & 39 & 7 & 43 & 2 \\
\hline 1103 & 5 & 91 & 2 & 83 & 2 & 57 & 11 & 67 & 2 & 53 & 2 & 51 & 7 \\
\hline 09 & 2 & 97 & 10 & 87 & 5 & 63 & 3 & 71 & 14 & 63 & 5 & 67 & 2 \\
\hline 17 & 2 & 1301 & 2 & 89 & 14 & 67 & 2 & 73 & 10 & 69 & 2 & 69 & 2 \\
\hline 23 & 2 & 03 & 6 & 93 & 2 & 69 & 2 & 77 & 2 & 81 & 3 & 73 & 3 \\
\hline 29 & 11 & 07 & 2 & 99 & 2 & 93 & 2 & 79 & 6 & 83 & 2 & 81 & 7 \\
\hline 51 & 17 & 19 & 13 & 1511 & 11 & 97 & 3 & 89 & 3 & 87 & 5 & 87 & 19 \\
\hline 53 & 5 & 21 & 13 & 23 & 2 & 99 & 3 & 1901 & 2 & 83 & 7 & 93 & 2 \\
\hline 63 & 5 & 27 & 3 & 31 & 2 & 1709 & 3 & 07 & 2 & 99 & 2 & 97 & 5 \\
\hline 71 & 2 & 61 & 3 & 43 & 5 & 21 & 3 & 13 & 3 & 2111 & 7 & 2309 & 2 \\
\hline 81 & 7 & 67 & 5 & 49 & 2 & 23 & 3 & 31 & 2 & 13 & 5 & 11 & 3 \\
\hline 87 & 2 & 73 & 2 & 53 & 3 & 33 & 2 & 33 & 5 & 29 & 3 & 33 & 2 \\
\hline 93 & 3 & 81 & 2 & 59 & 19 & 41 & 2 & 49 & 2 & 31 & 2 & 39 & 2 \\
\hline 1201 & 11 & 99 & 13 & 67 & 3 & 47 & 2 & 51 & 3 & 37 & 10 & 41 & 7 \\
\hline 13 & 2 & 1409 & 3 & 71 & 2 & 53 & 7 & 73 & 2 & 41 & 2 & 47 & 3 \\
\hline 17 & 3 & 23 & 3 & 79 & 3 & 59 & 6 & 79 & 2 & 43 & 3 & 51 & 13 \\
\hline 23 & 5 & 27 & 2 & 83 & 5 & 77 & 5 & 87 & 2 & 53 & 3 & 57 & 2 \\
\hline
\end{tabular}


Appendix 221

\begin{tabular}{|c|c|c|c|c|c|c|c|c|c|c|c|c|c|}
\hline$p$ & $g$ & $p$ & $g$ & $p$ & $g$ & $p$ & $g$ & $p$ & $g$ & $p$ & $g$ & $p$ & $g$ \\
\hline 2371 & 2 & 2617 & 5 & 2803 & 2 & 3061 & 6 & 3319 & 6 & 3541 & 7 & 3769 & 7 \\
\hline 77 & 5 & 21 & 2 & 19 & 2 & 67 & 2 & 23 & 2 & 47 & 2 & 79 & 2 \\
\hline 81 & 3 & 33 & 3 & 33 & 5 & 79 & 6 & 29 & 3 & 57 & 2 & 93 & 5 \\
\hline 83 & 5 & 47 & 3 & 37 & 2 & 83 & 2 & 31 & 3 & 59 & 3 & 97 & 2 \\
\hline 89 & 2 & 57 & 3 & 43 & 2 & 89 & 3 & 43 & 5 & 71 & 2 & 3803 & 2 \\
\hline 93 & 3 & 59 & 2 & 51 & 2 & 3019 & 6 & 47 & 2 & 81 & 2 & 21 & 3 \\
\hline 99 & 11 & 63 & 5 & 57 & 11 & 19 & 7 & 59 & 11 & 83 & 3 & 23 & 3 \\
\hline 2411 & 6 & 71 & 7 & 61 & 2 & 21 & 7 & 61 & 22 & 93 & 3 & 33 & 3 \\
\hline 17 & 3 & 77 & 2 & 79 & 7 & 37 & 3 & 71 & 2 & 3607 & 5 & 47 & 5 \\
\hline 23 & 5 & 83 & 2 & 87 & 5 & 63 & 3 & 73 & 5 & 13 & 2 & 51 & 2 \\
\hline 37 & 2 & 87 & 5 & 97 & 3 & 67 & 5 & 89 & 3 & 17 & 3 & 53 & 2 \\
\hline 41 & 6 & 89 & 19 & 2903 & 5 & 69 & 7 & 91 & 3 & 23 & 5 & 63 & 5 \\
\hline 47 & 5 & 93 & 2 & 09 & 2 & 81 & 7 & 3407 & 5 & 31 & 21 & 77 & 2 \\
\hline 59 & 2 & 99 & 2 & 17 & 5 & 87 & 2 & 13 & 2 & 37 & 2 & 81 & 13 \\
\hline 67 & 2 & 2707 & 2 & 27 & 5 & 91 & 11 & 33 & 5 & 43 & 2 & 89 & 11 \\
\hline 73 & 5 & 11 & 7 & 39 & 2 & 3203 & 2 & 49 & 3 & 59 & 2 & 3907 & 2 \\
\hline 77 & 2 & 13 & 5 & 53 & 13 & 09 & 3 & 57 & 7 & 71 & 13 & 11 & 13 \\
\hline 2503 & 3 & 19 & 3 & 57 & 2 & 17 & 5 & 61 & 2 & 73 & 5 & 17 & 2 \\
\hline 21 & 17 & 29 & 3 & 63 & 2 & 21 & 10 & 63 & 3 & 77 & 2 & 19 & 3 \\
\hline 31 & 2 & 31 & 3 & 69 & 3 & 29 & 6 & 67 & 2 & 91 & 2 & 23 & 2 \\
\hline 39 & 2 & 41 & 2 & 71 & 10 & 51 & 6 & 69 & 2 & 97 & 5 & 29 & 3 \\
\hline 43 & 5 & 49 & 6 & 99 & 17 & 53 & 2 & 91 & 2 & 3701 & 2 & 31 & 2 \\
\hline 49 & 2 & 53 & 3 & 3001 & 14 & 57 & 3 & 99 & 2 & 09 & 2 & 43 & 3 \\
\hline 51 & 6 & 67 & 3 & 11 & 2 & 59 & 3 & 3511 & 7 & 19 & 7 & 47 & 2 \\
\hline 57 & 2 & 77 & 3 & 19 & 2 & 71 & 3 & 17 & 2 & 27 & 3 & 67 & 6 \\
\hline 79 & 2 & 89 & 2 & 23 & 5 & 99 & 2 & 27 & 5 & 33 & 2 & 89 & 2 \\
\hline 91 & 7 & 91 & 6 & 37 & 2 & 3301 & 6 & 29 & 17 & 39 & 7 & & \\
\hline 93 & 7 & 97 & 2 & 41 & 3 & 07 & 2 & 33 & 2 & 61 & 3 & & \\
\hline 2609 & 3 & 2801 & 3 & 49 & 11 & 13 & 10 & 39 & 2 & 67 & 5 & & \\
\hline
\end{tabular}


This page is intentionally left blank 


\section{ANSWERS TO EXERCISES}

Here for some exercises we give the answers and for others we give hints, we give only a guide to the readers for reference.

\section{Exercise 1.1}

1. $l a \pm k a=(1 \pm k) a$.

2. $|k a|=|k||a| \geq|a|$, for $|k| \geq 1$.

3. Since $a=10 q+b$ and $5 \mid b$, then $5 \mid a$.

4. Among $a, b$, and $c$, there are two which are both even or odd. The sum and difference of these are both even.

5. Note $(2 n+1)^{2}-1=2 n \cdot 2(n+1)=4 n(n+1)$.

6. $n=3 q+r, r=0,1,2$. When $r=0$, then $3 \mid n$; when $r=1$, then $2 n=6 q+2$ and hence $3 \mid(2 n+1)$; when $r=2$, then $3 \mid(n+1)$.

7. $n(n-1)(2 n-1)=n(n-1)(n+1)+n(n-1)(n-2)$. We get the result from Example 2.

8. Note

$$
\begin{aligned}
& a^{n+1}-a(a-1) n-a \\
& =(a-1)\left(a^{n}+a^{n-1}+\ldots+a-n\right) \\
& =(a-1)\left\{\left(a^{n}-1\right)+\left(a^{n-1}-1\right)+\ldots+(a-1)\right\} \\
& =(a-1)^{2}\left(a^{n-1}+2 a^{n-2}+\ldots+n\right) .
\end{aligned}
$$

9. Since $n^{4}+4 n^{2}+11=n^{4}+4 n^{2}-5+16=\left(n^{2}+5\right)\left(n^{2}-1\right)+16$, when $n=2 m+1$, then $\left(n^{2}+5\right)\left(n^{2}-1\right)=8 m(m+1)\left(2 m^{2}+2 m+3\right)$, hence $16 \mid\left(n^{4} 4 m^{2}+11\right)$.

10. Let $n=2 m$, then $n(n+1)(n+2)=2 m(2 m+1)(2 m+2)=$ $4 m(2 m+1)(m+1)=4 m(m+1)\{(m+2)+(m-1)\}=4 m(m+$ 
$1)(m+2)+4 m(m+1)(m-1)$. From Example 2, the last two terms are both the multiples of 24 .

11. Let $s=2 a+3 b, t=9 a+5 b$. Eliminating $a$ we get $9 s-2 t=17 b$. If $17 \mid s$, then $17 \mid t$. The converse is also true.

12. $1112=(788)_{12}, 1112=(10001011000)_{2}$

13. As $d_{1}=1, d_{2}, \ldots, d_{k}=n$ are all positive factors of $n$, then $\frac{n}{d_{1}}, \frac{n}{d_{2}}, \ldots, \frac{n}{d_{k}}$ are also all positive factors of $n$. Hence $d_{1} d_{2} \cdots d_{k}$ $=\frac{n}{d_{1}} \frac{n}{d_{2}} \cdots \frac{n}{d_{k}}$, that is, $\left(d_{1} d_{2} \cdots d_{k}\right)^{2}=n^{2}$.

14. If $a$ is even, then

$$
\begin{aligned}
a^{n}= & a a^{n-1} \\
= & \left(a^{n-1}-a+1\right)+\left(a^{n-1}-a+3\right)+\ldots+\left(a^{n-1}-3\right) \\
& +\left(a^{n-1}-1\right)+\left(a^{n-1}+a-1\right)\left(a^{n-1}+a-3\right)+\ldots \\
& +\left(a^{n-1}+3\right)+\left(a^{n-1}+1\right)
\end{aligned}
$$

and the right-hand side is the sum of a consecutive odd integers. If $a$ is odd, then

$$
\begin{aligned}
a= & a^{n-1}+\left(a^{n-1}+2\right)+\left(a^{n-1}+4\right)+\ldots+\left(a^{n-1}+a-1\right) \\
& +\left(a^{n-1}-2\right)+\left(a^{n-1}-4\right)+\ldots+\left(a^{n-1}-a+1\right)
\end{aligned}
$$

and the right-hand side is also the sum of $a$ consecutive odd integers.

15. Use mathematical induction. $n=1$ is trivial. Assume this result is true for $n$, i.e., there are $n$ consecutive integers $1+k$ and $m_{k}^{2} \mid(1+k), k=1, \ldots, n$. Let $m=m_{1} m_{2} \cdots m_{n}$. Then

$$
\begin{gathered}
m_{k}^{2} \mid\left\{m^{2}\left(m^{2}-2\right)(1+n+1)+1+k\right\} \\
(m+1)^{2} \mid\left\{m^{2}\left(m^{2}-2\right)(1+n+1)+1+n+1\right\} \\
=\left(m^{2}-1\right)^{2}(1+n+1)
\end{gathered}
$$

and hence

$$
m^{2}\left(m^{2}-2\right)(1+n+1)+1+k, \quad k=1, \ldots, n+1,
$$


are the required $n+1$ consecutive integers.

16. Let the least integer be $a$, the common difference be $d$. Then

$$
\begin{aligned}
& \qquad \begin{array}{l}
a^{2}+(a+d)^{2}+\ldots+(a+8 d)^{2}=x^{2} \\
9 a^{2}+72 a d+204 d^{2}=x^{2}, \\
3 a^{2}+24 a d+68 d^{2}=3 k^{2}, \quad x=3 k
\end{array} \\
& \text { that is, } \begin{array}{l}
\text { or } \quad d \text { is the multiple of 3, i.e., } d=3 d \text {. Thus } \\
\text { Since }(3,68)=1, \\
k^{2}=a^{2}+24 a d^{\prime}+204 d^{\prime 2}=\left(a+14 d^{\prime}\right)^{2}+\left(8 d^{\prime 2}-4 a d^{\prime}\right) .
\end{array}
\end{aligned}
$$

Putting

$$
8 d^{\prime 2}=4 a d^{\prime}, \text { or } a=2 d^{\prime} \text { and } d^{\prime}=1,
$$

we get the required system of solutions:

$2, \quad 5,8,11,14,17,20,23,26$.

\section{Exercise 1.2}

1. $(24871,3468)=17,(120,504,882)=6,[135,513,3114]=887490$.

2. As $n^{3}-n=(n-1) n(n+1)$ is the product of three consecutive integers, it is a multiple of 3 . Again as $n$ is an odd integer, $n-1$ and $n+1$ are both even and one of them is a multiple of 4 . Hence $(n-1)(n+1)$ is a multiple of 8 . Thus $n^{3}-n$ is the multiple of $3 \cdot 8=24$.

3. Let $b$ be the common divisor, $d$ the greatest common divisor. As $[b, d]=c$, then $d \leq c$. Again as $b\left|a_{i}, d\right| a_{i}$, then $c \mid a_{i}$, and hence $c$ is the common divisor of $a_{1}, \ldots, a_{n}$. But $d$ is the greatest divisor, hence $c \leq d$. Thus $c=d$, and $b \mid d$.

4. If $\left(a_{1}, a_{2}\right)=1$, then $\left[a_{1}, a_{2}\right]=a_{1} a_{2}$. Similarly,

$$
\left[a_{1}, a_{2}, a_{3}\right]=\left[\left[a_{1}, a_{2}\right], a_{3}\right]=\left[a_{1} a_{2}, a_{3}\right]=a_{1} a_{2} a_{3} .
$$


5. Since any integer of the sequence $a, 2 a, \ldots, b a$ is a multiple of $a$, then a multiple of $b$ is also a multiple of the least common multiple $[a, b]$ of $a$ and $b$. Hence the number of multiples of $b$ in the sequence is $\frac{b a}{[a, b]}=(a, b)$.

6. If $x=8 a, y=8 b,(a, b)=1$, as $(x, y)[x, y]=x y$, then $8 \cdot 64=$ 64ab. That is, $a b=8$. Thus $a=1$ or $a=8$. Therefore $x=8$, $y=64$ or $x=64, y=8$.

7.

$$
\begin{aligned}
(a+b,[a+b]) & =\frac{((a+b)(a, b), a b)}{(a, b)} \\
& =\frac{\left(a^{2}, a b, b^{2}\right)}{(a, b)}=(a, b)
\end{aligned}
$$

8.

$$
\begin{aligned}
([a, b], c) & =\frac{[a, b] c}{[[a, b], c]}=\frac{[a, b] c}{[a, b, c]} \\
& =\frac{(a b, b c, c a)}{(a, b)}
\end{aligned}
$$

also

$$
[(a, c),(b, c)]=\frac{(a, c)(b, c)}{((a, c),(b, c))}=\frac{(a, c)(b, c)}{(a, b, c)}
$$

As

$$
\frac{(a b, b c, c a)}{(a, b)}=\frac{(a, c)(b, c)}{(a, b, c)}
$$

that is

$$
(a, b)(b, c)(c, a)=(a, b, c)(a b, b c, c a)
$$

9. $(a, b)^{2}=(a, b)(a, b)=(a(a, b), b(a, b))=\left(a^{2}, a b, b^{2}\right)=\left(a^{2}, b^{2}\right)$. Similarly $[a, b]^{2}=[a, b][a, b]=\left[a^{2}, a b, b^{2}\right]=\left[a^{2}, b^{2}\right]$.

10. As in the previous exercise, expanding the two sides we obtain the result. 
11. Let $(a-b, a+b)=d$. Then $a-b=k d, a+b=l d$. Hence $2 a=(k+l) d, 2 b=(k-l) d$. Thus $2=(2 a, 2 b)=d(k+l, k-l)$, or $d \mid 2$.

12. Suppose $a, b$ are both positive integers. Since $[a, b](a, b)=a b$, letting $[a, b]=a k, s=-a /(a, b)$, we get $a k+b s=0$. Also since $k=a b / a(a, b)=b /(a, b)$, then $(k, s)=1$ and hence $h s+(-k) r=$ 1 .

13. Let $\left(2^{m}-1,2^{m}+1\right)=d$. Then $2^{m}=k d+1,2^{n}=l d-1, k l>0$. Thus $2^{m n}=(k d+1)^{n}=t d+1,2^{n m}=(l d-1)^{m}=s d-1$, where $(s-t) d=2$. Consequently $d \mid 2$, that is, $d=1$ or 2 . But as $2^{m}-1$, $2^{n}+1$ are both odd integers, hence $d=1$.

14. Let $(21 n+4,14 n+3)=d$. Since $d \mid(21 n+4)$ and $d \mid(14 n+3)$,

$$
d \mid(3(14 n+3)-2(21 n+4))=1, \text { or } d=1 .
$$

15. $1=\left(a_{1}+a_{2}, a_{1} b_{2}-a_{2} b_{1}\right)=\left(a_{1}+a_{2}, a_{1} b_{2}+a_{1} b_{1}\right)=\left(a_{1}+a_{2}, b_{1}+\right.$ $\left.b_{2}\right)$.

16. Let $d_{0}=a x_{0}+b y_{0}$ be the least positive integer of $f(x, y)$. Evidently $d \mid d_{0}$ hence $d \leq d_{0}$. Also from Theorem 6 , Sec. 1.2 we get $d=a x+b y$. As $d_{0}$ is the minimum $d \geq d_{0}$. Hence $d=d_{0}$.

\section{Exercise 1.3}

1. $T(2160)=40, S(2160)=7440$.

2. As $p \mid r ! c_{p}^{r}$, and $r<p$, hence $p \mid c_{p}^{r}$.

3. Let $(x, y)=d$, that is, $x=d x_{1}, y=d y_{1},\left(x_{1}, y_{1}\right)=1$. Then $x_{1}^{2}+y_{1}^{2}=a x_{1} y_{1}$ and hence $x_{1}\left|y_{1}^{2}, y_{1}^{2}\right| x_{1}^{2}$. Thus $x_{1}=y_{1}=1$, that is $a=2$.

4. 1) If $3\left|a^{2}, 3\right| b^{2}$, then $3|a, 3| b$. 2) Suppose $3 \nmid a^{2}, 3 \not b^{2}$. Let $a=$ $3 k+r, b=3 l+s$. Then $a^{2}+b^{2}=9 k^{2}+6 k r+9 l^{2}+6 l s+r^{2}+s^{2}$, and hence $3 \mid\left(r^{2}+s^{2}\right)$. But when $r=1,2$, then $r^{2}=1,4$. Therefore $r^{2}+s^{2}=2,5,8$. They are not multiples of 3 , which is impossible. 
5. Since $n^{4}+4=\left(n^{2}+2\right)^{2}-(2 n)^{2}=\left(n^{2}+2 n+2\right)\left(n^{2}-2 n+2\right)$, if $n>t$ then $n^{2}-2 n+2 \geq 2$; hence $n^{4}+4$ is a composite number.

6. Since $T(a)=\prod_{i=1}^{k}\left(a_{i}+1\right)=10$, then $\left(a_{1}+1\right)\left(a_{2}+1\right)=1 \cdot 10$ or $\left(a_{1}+1\right)\left(a_{2}+1\right)=2 \cdot 5$. In the former case $a_{1}=0, a_{2}=9$ and hence $a=p_{1}^{9}$; in the latter case $a_{1}=1, a_{2}=4$ and hence $a=p_{1} p_{2}^{4}$. Therefore, the required least positive integer is $a=3 \cdot 2^{4}=48$.

7. Since $s(a)=\frac{p_{1}^{a_{1}+1}-1}{p_{1}-1} \cdot \frac{p_{2}^{a_{2}+1}-1}{p_{2}-1}=15$, then $p_{1}=2, a_{1}=3$, or $a=2^{3}=8$.

8. We have $a^{\frac{1}{2} T(a)}=64=2^{6}$, or $a^{T(a)}=2^{12}$. Set $a=2^{a_{1}}$. Since $T(a)=a_{1}+1,2^{a_{1}\left(a_{1}+1\right)}=2^{12}, a_{1}\left(a_{1}+1\right)=12$. Thus $a=3$ or $a=-4$, hence $a=2^{3}=8$.

9. Since $a^{\frac{1}{2} T(a)-1}=a$, or $a^{\frac{1}{2} T(a)}=a^{2}$, then $T(a)=4$. Thus $\left(a_{1}+\right.$ 1) $\left(a_{2}+1\right)=4$, and hence $a_{1}=3, a_{2}=0$ or $a_{1}=1$, that is, $a=p^{3}$ or $a=p q$.

10. Let $b \geq c$. If $a \geq b \geq c$, then $\max (\min (a, b), \min (a, c))=$ $\max (b, c)=b, \min (a, \max (b, c))=\min (a, b)=b$. Hence the given equation exists. If $b \geq a \geq c$ or $b \geq c \geq a$, the proof is similar.

11. By the hypothesis $a+b=\frac{a b}{c}$, as $a, b$ are both integers, then $c=q r$, where $q|a, r| b$. From $(a, b, c)=1$, we get $(q, r)=1$. Set $a=m q, b=p r$. Then $m p+p r=\frac{m q \cdot p r}{q r}=m p$, and hence $m \mid p r$. Also from $(a, b, c)=1$, then $(m, r)=1$, and hence $m \mid p$. Similarly, we have $p \mid m q,(p, q)=1$, and hence $p \mid m$. Consequently $p=m$. By substituting, we get $p(p+r)=p^{2}$, or $p=q+r$. Therefore $a+b=p q+p r=p(q+r)=p^{2}, a-c=p q-q r=q^{2}, b-c=r^{2}$.

12. For instance, $a$ can be taken as

$$
a=2 \cdot 3 \cdots(k+1) t+2, \quad t=1,2, \ldots
$$

13. Let $p_{1}, \ldots, p_{k}$ be primes not greater then $n$ and $q=p_{1} \cdots p_{k}-1$. Evidently $q$ has a prime factor $p$ different from $p_{i}$, thus $p>n$ and $p<q<n !-1<n !$. 
14. Suppose $p_{1}, p_{2} \ldots, p_{r}$ are primes of the form $3 n+2$. Put $a=$ $3 p_{1} p_{2} \ldots p_{r}-1=q_{1} q_{2} \ldots q_{t}$, where $q_{i}$ are primes. Clearly, $q_{i}$ are different from $3, p_{1}, p_{2} \ldots, p_{r}$. If all $q_{i}$ are of the form $3 n+1$, then their product $a$ is also of the form $3 n+1$. This contradicts with $a=3 p_{1} p_{2} \ldots p_{r}-1$, Hence at least one $q_{i}$ is of the form $3 n+2$.

15. Prove by contradiction. Assume $m=a b, 1<a<m$, then $n_{m}=$ $1+10+\ldots+10^{m-1}=\frac{10^{m}-1}{9}=\frac{10^{a b}-1}{9}$. Since $\left(10^{a}-1\right) \mid\left(10^{m}-1\right)$, $\frac{10^{a}-1}{9} \mid \frac{10^{m}-1}{9}=n_{m}$. But as $1<\frac{10^{a}-1}{9}<n_{m}$, this is impossible since $n_{m}$ is assumed to be prime.

16. From $945=3^{3} \cdot 5 \cdot 7$, we get $S(945)=\left(1+3+3^{2}+3^{3}\right)(1+5)(1+7)$; hence $S(945)>2 \cdot 945=1890$. Let $n=945 m, 2 \nmid m,(945, m)=1$ then $s(n)=s(945 \cdot m)=s(945) s(m) \geq s(945) m \geq 2 \cdot 945 m=2 n$.

17. As $p-1=q+1, p^{p}+q^{q}=\left(p^{p}-1\right)+\left(q^{q}+1\right), p^{p}-1=$ $(p-1)\left(p^{p-1}+\ldots+p+1\right)=(p-1)(2 m+1), q^{q}+1=(q+1)(2 n+1)=$ $(p-1)(2 n+1)$, we have

$$
p^{p}+q^{q}=2(p-1)(m+n+1)=(p+q)(m+n+1) .
$$

18. 1) They are 9 in number.

2) When $n=2 k$, there are $9 \cdot 10^{k-1}$ in number; when $n=2 k-1$, there are $9 \cdot 10^{k-1}$ in number.

3) $a b b a=a+10 b+10^{2} b+10^{3} a \equiv a-b+b-a \equiv 0(\bmod 11)$.

\section{Exercise 1.4}

1. The Fermat numbers not greater than $F_{n}$ are $F_{0}, F_{1}, \ldots, F_{n-1}$ and every such number has a prime factor. By Theorem 5 , these factors are different from one another.

2. $\left(M_{p}, M_{2}\right)=1, M_{2}=3 .\left(M_{p}, M_{3}\right)=1, M_{3}=7$.

3. If $n=p_{1} \ldots p_{k}$, the $p_{i}$ 's being primes, and $p_{1}<p_{2}<\ldots<p_{k}$, then $S(n)=\left(1+p_{1}\right) \ldots\left(1+p_{k}\right)=2 n$, or $\left(1+p_{1}\right) \ldots\left(1+p_{k}\right)=$ 
$2 p_{1} \ldots p_{k}$. When $k=1$, then $1+p_{1}=2 p_{1}$ and hence $p_{1}=1$, which is impossible. When $k \geq 2$, if $n$ is odd, then $p_{i}$ 's are all odd and hence $4 \mid S(n)$, or $2 \mid n$, which is also impossible. Thus $n$ is even. Therefore when $k=2$, then $n=6$. But when $k=3$ then $S(n)=$ $3\left(1+p_{2}\right)\left(1+p_{3}\right)=2 \cdot 2 p_{2} p_{3}$, which is clearly impossible. When $k>3$, since $8 \mid S(n)$, then $4 \mid n$. This contradicts the hypothesis that $n$ has no square factor. Therefore we can only have $n=6$.

4. When $a>2$, then $a-1$ is a factor of $a^{2}-1$ which contradicts the hypothesis. When $n$ is composite $n=n_{1} \cdot n_{2}$, then $\left(a^{n_{1}}-\right.$ 1) $\mid\left(a^{n}-1\right)$, which also contradicts the hypothesis.

\section{Exercise 1.5}

1. As $5(1000 !)=200+40+8+1=249, \frac{1000}{2}=500$ hence there are 249 zeros in 1000 !.

2. Primes not greater than 30 are the following 10 integers. 2, 3, $5,7,11,13,17,19,23,29$. Since $2(30 !)=26,3(30 !)=14$, $5(30 !)=7,7(30 !)=4,11(30 !)=2,13(30 !)=2,17(30 !)=1$, $19(30 !)=1,23(30 !)=1,29(30 !)=1$, hence $30 !=2^{26} \cdot 3^{14} \cdot 5^{7}$. $7^{4} \cdot 11^{2} \cdot 13^{2} \cdot 17 \cdot 19 \cdot 23 \cdot 29$.

3. $\left[\frac{545}{13}\right]=41,\left[\frac{175}{13}\right]=13$, the required number $=41-13=28$.

4. $m=2^{r}-1,\left[\frac{m}{2}\right]=2^{r-1}-1,\left[\frac{m}{2^{2}}\right]=2^{r-2}-1, \ldots,\left[\frac{m}{2^{r-1}}\right]=2-1=1$. Hence

$2\left(\left(2^{r}-1\right) !\right)=2^{r-1}+2^{r-2}+\ldots+2+1-r=\frac{2^{r}-1}{2-1}-r=2^{r}-r-1$.

5. $\left[\frac{n}{3}\right]+\left[\frac{n}{3^{2}}\right]+\ldots=7$. When $n=18,3(n !)=8$; when $n=17$, $3(n !)=6$. Hence such a number $n$ does not exist.

6. As $[x] \leq x<[x]+1,[x]+a \leq x+a<[x]+a+1$ and hence $[x+a]=[x]+a$.

7. As $x=[x]+r, y=[y]+s$, we have $x-y=[x]-[y]+r-s$, $r-s<1$. When $r-s \geq 0,[x-y]=[x]-[y]$; when $r-s<0$, $[x-y]=[x]-[y]-1$. 
8. Let $n=n_{1}+n_{2}+\ldots+n_{k}$. Since

$$
\frac{n}{p^{i}}=\frac{n_{1}}{p^{i}}+\frac{n_{2}}{p^{i}}+\ldots+\frac{n_{k}}{p^{i}}
$$

then

$$
\left[\frac{n}{p^{i}}\right] \geq\left[\frac{n_{1}}{p^{i}}\right]+\left[\frac{n_{2}}{p^{i}}\right]+\ldots+\left[\frac{n_{k}}{p^{i}}\right]
$$

Hence

$$
\sum\left[\frac{n}{p^{i}}\right] \geq \sum\left[\frac{n_{1}}{p^{i}}\right]+\sum\left[\frac{n_{2}}{p^{i}}\right]+\ldots+\sum\left[\frac{n_{k}}{p^{i}}\right] .
$$

That is, the greatest power of $p$ in $n$ ! is not less than that in $n_{1} ! n_{2} ! \ldots n_{k} !$. Hence it is an integer.

9. For positive integer $r(1 \leq r \leq b-1)$, let $\frac{r}{b} a=n+\frac{a^{\prime}}{b}\left(0<a^{\prime}<b\right)$, then $\frac{a^{\prime}}{b}<1$. Hence

$$
\begin{aligned}
{\left[-\frac{r}{b} a\right] } & =-(n+1)=-\left[\frac{r}{b} a\right]-1 \\
{\left[\frac{b-r}{b} a\right] } & =\left[a-\frac{r}{b} a\right]=a+\left[-\frac{r}{b} a\right] \\
& =a-\left[\frac{r}{b} a\right]-1=a-1-\left[\frac{r}{b} a\right] .
\end{aligned}
$$

Thus

$$
\sum_{r=1}^{b-1}\left[\frac{r}{b} a\right]=\sum_{r=1}^{b-1}\left[\frac{b-r}{b} a\right]=(b-1)(a-1)-\sum\left[\frac{r}{b} a\right] .
$$

Therefore

$$
\sum_{r=1}^{b-1}\left[\frac{r}{b} a\right]=\frac{(b-1)(a-1)}{2}
$$

10. For $\left[(3 m+1) \cdot \frac{4}{3}\right]=\left[4 m+1+\frac{1}{3}\right]=4 m+1$. 
11. Similar to theorem $7, \sum_{d \mid n}|\mu(d)|=1+k+\left(\frac{k}{2}\right)+\left(\frac{k}{3}\right)+\ldots+1^{k}=$ $(1+1)^{k}=2^{k}$.

\section{Exercise 2.1}

1. $a=m k+b$, then $(a, m)=(m k+b, m)=(b, m)$.

2. When $a \equiv 0(\bmod 3)$ or $a \equiv 2(\bmod 3), a^{2}+a+1 \equiv 1(\bmod 3)$. When $a \equiv 1(\bmod 3), a^{2}+a+1 \equiv(\bmod 3)$.

3. Since $504=7 \cdot 8 \cdot 9$, when $n \equiv 0, \pm 1, \pm 2, \pm 3(\bmod 7)$, we have

$$
n^{3} \equiv 0, \pm 1(\bmod 7), \quad n^{9} \equiv 0, \pm 1(\bmod 7) \text {. }
$$

Hence $n^{9}-n^{3} \equiv 0(\bmod 7)$, or $7 \mid\left(n^{9}-n^{3}\right)$. Similarly when $n=0, \pm 1, \pm 2, \pm 3, \pm 4(\bmod 8)$ and $n \equiv 0, \pm 1, \pm 2, \pm 3, \pm 4$ $(\bmod 9)$, we have $n^{9}-n^{3} \equiv 0(\bmod 8)$ and $n^{9}-n^{3} \equiv 0(\bmod 9)$, that is

$$
8\left|\left(n^{9}-n^{3}\right), \quad 9\right|\left(n^{9}-n^{3}\right) .
$$

Hence $504 \mid\left(n^{9}-n^{3}\right)$.

4. For modulo $11,10 \equiv-1,10^{2} \equiv 1,10^{3} \equiv-1, \ldots$ Hence $n=$ $a_{0}+a_{1} 10+a_{2} 10^{2}+\ldots \equiv a_{0}-a_{1}+a_{2}-\ldots$ or $\left(a_{0}+a_{2}+\ldots\right)-$ $\left(a_{1}+a_{3}+\ldots\right) \equiv 0$.

6. $1000 \equiv-1(\bmod 13)$. As in Example 3 we get

$$
\left(a_{0}+a_{2}+\ldots\right) \equiv\left(a_{1}+a_{3}+\ldots\right)(\bmod 13) .
$$

Now $637693=693+637 \cdot 1000, a_{0}-a_{1}=693-637=56 \not \equiv$ $0(\bmod 13)$. Hence it is not divided by 13 .

7. Since for modulo $9, a \equiv 8, b \equiv 3, c \equiv 5$, and $a b \equiv 24 \not \equiv 5$. Hence the result is wrong.

8. Since $g \equiv 0(\bmod d), g^{m} \equiv 0\left(\bmod d^{m}\right)$. Hence

$$
\begin{aligned}
\left(c_{m} c_{m-1} \ldots c_{1} c_{0}\right)_{g} & \equiv c_{m-1} g^{m-1}+\ldots+c_{1} g \pm c_{0} \\
& \equiv\left(c_{m-1} \ldots c_{1} c_{0}\right)_{g}\left(\bmod d^{m}\right)
\end{aligned}
$$


9. From the binomial theorem

$$
(a+b)^{p}=a^{p} \frac{p}{1} a^{p-1} b+\frac{p(p-1)}{2} a^{p-2} b^{2}+\ldots+\frac{p}{1} a b^{p-1}+b^{p},
$$

we get $(a+b)^{p}-\left(a^{p}+b^{p}\right)=p\left(a^{p-1} b+\frac{p-1}{2} a^{p-2} b^{2}+\ldots a b^{p-1}\right)$, and hence $(a+b)^{p}-\left(a^{p}+b^{p}\right) \equiv 0(\bmod p)$.

\section{Exercise 2.2}

1. The proof can be obtained from the definition of a complete system of residues.

2. Since for modulo $p, a^{p-1} \equiv 1, p-1 \equiv-1,(p-1)^{a} \equiv(-1)^{a}$. Hence when $a$ is odd, $a^{p-1}+(p-1)^{a} \equiv 0$; when $a$ is even, $a^{p-1}-(p-1)^{a} \equiv 0$.

3. As $2 n(2 n+2)=4 n(n+1)$, we have $2 \mid n(n+1)$, or $8 \mid 2 n(2 n+2)$. Next, $p^{4}-1=(p-1)(p+1)\left(p^{2}+1\right), 240=2^{4} \cdot 3 \cdot 5$. As $8 \mid(p-1)(p+1), p^{2} \equiv 1(\bmod 3)$ or $3 \mid(p-1)(p+1)$, and $2 \mid\left(p^{2}+1\right)$. As $p^{4} \equiv 1(\bmod 5)$ or $5 \mid\left(p^{4}-1\right)$, we have $240 \mid\left(p^{4}-1\right)$.

4. $p^{2}-q^{2}=p^{2}-1-\left(q^{2}-1\right)$. Since $3\left|\left(p^{2}-1\right), 8\right|\left(p^{2}-1\right)$, we have $24 \mid\left(p^{2}-1\right)$. Similarly, $24 \mid\left(q^{2}-1\right)$, hence $24 \mid\left(p^{2}-q^{2}\right)$.

5. $p^{6}-1=\left(p^{2}-1\right)(\ldots), 168=2^{3} \cdot 3 \cdot 7$. Since $p^{6} \equiv 1(\bmod 7)$ or $7\left|\left(p^{6}-1\right), 3\right|\left(p^{2}-1\right), 8 \mid\left(p^{2}-1\right)$, we have $168 \mid\left(p^{6}-1\right)$.

6. $a^{p-1} \equiv 1(\bmod p), a^{2(p-1)} \equiv 1(\bmod p)$ and $a^{2(p-1)}=a^{q-1} \equiv$ $1(\bmod q)$. Let $p-1=2 t$. Then $a^{2(p-1)}-1=\left(a^{t}\right)^{4}-1=$ $\left(a^{2 t}-1\right)\left(a^{2 t}+1\right)$, and hence $8\left|\left(a^{2 t}-1\right), 2\right|\left(a^{2 t}+1\right)$. Therefore $a^{2(p-1)} \equiv 1(16 p q)$.

7. As $a^{m-1}=\left(a^{\frac{m-1}{2}}\right)^{2} \equiv(-1)^{2} \equiv 1(\bmod m)$, the result follows from Theorem 7 .

8. As $p^{q-1} \equiv 1(\bmod q), p^{q-1}+q^{p-1} \equiv 1(\bmod q)$. Similarly, $q^{p-1}+$ $p^{q-1} \equiv 1(\bmod p)$, and therefore $p^{q-1}+q^{p-1} \equiv 1$ $(\bmod p q)$. 
9. As $b^{p}-b=b\left(p^{p-1}-1\right)=b\left(\left(b^{2}\right)^{\frac{p-1}{2}}-1\right)=b\left(b^{2}-1\right)\left(\left(b^{2}\right)^{\frac{p-1}{2}-1}+\right.$ $\ldots+1)$, hence $b\left(b^{p}-1\right) \mid\left(b^{2}-b\right)$. But as $6 \mid b\left(b^{2}-1\right)$, hence $6 \mid\left(b^{p}-\right.$ $b)$. Again, since $b^{p}-b \equiv 0(\bmod p), 6 p \mid\left(b^{p}-b\right)$ or $b^{p}-b \equiv$ $0(\bmod 6 p) ;$ similarly, $a^{p}-a \equiv 0(\bmod 6 p)$. Thus $a\left(b^{p}-b\right) \equiv$ $b\left(a^{p}-a\right)(\bmod 6 p)$. Therefore, $a b^{p} \equiv b a^{p}(\bmod 6 p)$.

10. Let $m=\phi(b), n=\phi(a)$. From $(a, b)=1$ we get $a^{m} \equiv 1(\bmod b)$, $b^{n} \equiv 1(\bmod a)$. Thus $a^{m}+b^{n} \equiv b^{n} \equiv 1(\bmod a), a^{m}+b^{n} \equiv$ $a^{m} \equiv 1(\bmod b)$. Hence $a^{m}+b^{n} \equiv 1(\bmod a b)$.

\section{Exercise 2.3}

1. As $\phi(60)=16,1956=122 \cdot 16+4$, hence $13^{1956} \equiv 13^{4}=(169)^{2}=(-11)^{2}=121 \equiv 1(\bmod 60)$.

2. Let $m=p_{1}^{m_{1}} \ldots p_{r}^{m_{r}}$. Then $\phi(m)=p_{1}^{m_{1}-1} \ldots p_{r}^{m_{r}-1}\left(p_{1}-1\right) \ldots$ $\left(p_{r}-1\right)$. When $\phi(m)$ is odd, then $p_{i}$ are not odd and hence $p_{1}=2, m_{1}=1$ or $m=2$. Besides this we also have $m=1$.

3. The proof is similar to that in the previous exercise.

5. Let $x=p_{1}^{a_{1}} \ldots p_{r}^{a_{r}}$. Then $\phi(x)=p_{1}^{a_{1}-1} \ldots p_{r}^{a_{r}-1}\left(p_{1}-1\right) \ldots\left(p_{r}-\right.$ $1)=2(2 n-1)$. Evidently $r=1$, or $p_{1}^{a_{1}-1}\left(p_{1}-1\right)+2(2 n-1)$. Since for modulo $4, p_{1}-1 \not \equiv 0, p_{1}-1 \not \equiv 1, p_{1}-1 \not \equiv 3$ we have $p_{1}-1 \equiv 2$. Therefore $p_{1}=4 k-1$; thus $x=p_{1}^{a_{1}}$ or $x=2 p_{1}^{a_{1}}$.

6. If $\frac{m}{n}$ is reduced proper fraction, then $m$ is a number in the reduced residue system of $n$.

7. $\frac{1}{2}, \frac{1}{3}, \frac{2}{3}, \ldots, \frac{m_{1}}{n}, \ldots, \frac{m_{\varphi(n)}}{n}$ are $\phi(2)+\phi(3)+\ldots+\phi(n)$ in number.

9. Let $p_{1}, \ldots, p_{k}$ be the different prime factors of $m$. Since $\phi(m)=$ $m \prod_{i=1}^{k} \frac{p_{i}-1}{p_{i}}$, then $\prod \frac{p_{i}-1}{p_{i}}=\frac{1}{p}$, or $p \prod\left(p_{i}-1\right)=p_{1} \ldots p_{k}$. Let $p_{1}$ be the greatest of $p_{1}, \ldots, p_{k}$, then $p_{1} \mid p$ and hence $p_{1}=p$. Thus $\Pi\left(p_{i}-1\right)=p_{2} \ldots p_{k}$. As $p_{i}-1$ is even, the number of odd primes among $p_{1}, \ldots, p_{k}$ cannot exceed 1 . Hence when $p_{1}, \ldots, p_{k}$ all are not odd, $p_{1}=2$. When among $p_{1} \ldots p_{k}$ there exists an 
odd integer, there also exists an even integer. This time we have $p_{1}=3, p_{2}=2$. Therefore, the required numbers are

$$
p=2, m=2^{k} \text { or } p=3, m=2^{k} 3^{l},
$$

where $k, l$ are any positive integers.

10. Let $\phi(y)=r$, when $x>2$, then $r=\phi\left(x^{p(y)}\right)>x^{\phi(y)-1}=x^{r-1}$, or $r>x^{r-1}, x>2$. It is impossible. Hence $x \leq 2$. Therefore the required solutions are

$$
\left\{\begin{array} { l } 
{ x = 1 } \\
{ y = 1 }
\end{array} \quad \left\{\begin{array} { l } 
{ x = 2 } \\
{ y = 2 }
\end{array} \quad \left\{\begin{array}{l}
x=2 \\
y=4
\end{array} .\right.\right.\right.
$$

\section{Exercise 3.1}

1. 1) $\left\{\begin{array}{l}x=-3+7 t \\ y=5-8 t\end{array}\right.$

2) $\left\{\begin{array}{l}x=4-9 t_{1}-10 t_{2} \\ y=2-4 t_{1}-5 t_{2} \\ z=2-t_{2}\end{array}\right.$

3) $\left\{\begin{array}{l}x=-18-54 c+21 b+5 a \\ y=6+18 c-7 b-2 a \\ z=1+3 c-b \\ t=3-c\end{array}\right.$

2. 1) It has 5 systems of solutions where $x=11,31,51,71,91$.

2) From $x(x+y)=6$, we get $x=1, y=5$, and $x=2, y=1$.

3) Reducing the given equation to the form $y=2+\frac{4}{x-1}$, we get $x-1=1,2,4$, that is, $x=2,3,5$.

3. Eliminating $x$, we get $2 y-z=3$. Solving, we obtain $y=2+$ $t, z=1+2 t$. Substituting in the given equation, we get $x=$ $3-8 t$.

4. Eliminating $z$ by substituting $z=3-(x+y)$ in the first equation, we have

$$
8-3 x(3-x)-3 y(3-x)+x y(3-x)+y^{3}(3-x)=0 .
$$


Hence $(3-x) \mid 8$. Thus $3-x= \pm 1, \pm 2, \pm 4, \pm 8$, that is, it is possible that $x=-5,-1,1,2,4,5,7,11$. If $x=-5$, from the given equation we have $y^{3}+z^{3}=128, y+z=8$. Solving them we get a system of solutions $(-5,4,4)$. Similarly, for $x=-1,1,2,4,5,7,11$, we get 3 solutions $(1,1,1)$, $(4,-5,5),(4,4,-5)$. These 4 solutions are the required solutions.

5.

\begin{tabular}{c|rrrr} 
cock & 0 & 4 & 8 & 12 \\
\hline hen & 25 & 18 & 11 & 4 \\
\hline chicken & 75 & 78 & 81 & 84
\end{tabular}

6. Let $4 k+1$ be a number containing 2 digits. Since $10 \leq 4 k+1<$ 100 , or $2 \frac{1}{4}<k<24 \frac{3}{4}$, there are 22 integer values between 3 and 24 that $k$ can take. Thus we get 22 integers which all contain 2 digits and form an arithmetic progression whose sum is 1210 .

7. From $x+100=a^{2}, x+168=b^{2}$, we get $68=b^{2}-a^{2}=(b+$ $a)(b-a)$. Since 68 is even and $68=34 \cdot 2, b+a=34, b-a=2$, that is, $b=18, a=16$. Hence the required number is $x=156$.

8. Let $(x, y)=d, x=d x^{\prime}, y=d y^{\prime}$. Then $x^{\prime 2}+y^{\prime 2}=a x^{\prime} y^{\prime}$, hence $x^{\prime}\left|y^{\prime 2}, y^{\prime}\right| x^{\prime 2}$, or $x^{\prime}=1, y^{\prime}=1$; thus $a=2$.

9. $a^{2} x y+a b x+a c y+a d=0$ can be written as $(a x+c)(a y+b)=$ $b c-a d$. If $b c-a d \neq 0$, then $a x+c$ is a factor of $b c-a d$ and $x$ has only finite integer solutions. Similarly, $y$ also has only finite integer solutions. Now consider the case when $b c-a d=0$. If $a \mid c$, then $x=\frac{c}{a}, y$ being any integer, are solutions. Thus there exists an infinite system of solutions; if $a \mid b$ there also exists an infinite number of solutions.

10. Let $\left(x_{0}, y_{0}, z_{0}\right)$ be a system of solutions. From $a x_{0}+b y_{0}+c z_{0}=$ $k, a x+b y+c z=k$, we get $d\left\{a_{1}\left(x-x_{0}\right)+b_{1}\left(y-y_{0}\right)\right\}=-c\left(z-z_{0}\right)$. Since $(d, c)=1$, there exists an integer $t_{2}$ such that $z=z_{0}+d t_{2}$. Thus we have $a_{1}\left(x-x_{0}\right)+b_{1}\left(y-y_{0}\right)=-c t_{2}$. Since $a_{1} u_{1}+b_{1} u_{2}=$ 
1 , then $a_{1}\left(-u_{1} c t_{2}\right)+b_{1}\left(-u_{2} c t_{2}\right)=-c t$. Therefore there exists an integer $t_{1}$ such that $x=x_{0}+b_{1} t_{1}-u_{1} c t_{2}, y=y_{0}-a_{1} t_{1}-u_{2} c t_{2}$.

\section{Exercise 3.2}

1.

\begin{tabular}{r|rrrrrrrr}
$\mathrm{b}$ & 1 & 1 & 1 & 2 & 2 & 2 & 3 & 4 \\
$\mathrm{a}$ & 2 & 4 & 6 & 3 & 5 & 7 & 4 & 5 \\
\hline $\mathrm{x}$ & 3 & 15 & 35 & 5 & 21 & 45 & 7 & 9 \\
$\mathrm{y}$ & 4 & 8 & 12 & 12 & 20 & 28 & 24 & 40 \\
$\mathrm{z}$ & 5 & 17 & 37 & 13 & 29 & 53 & 25 & 41
\end{tabular}

2. Let $x-y=a^{2}, y-z=b^{2}, x-y=c^{2}$. Then $a^{2}+b^{2}=c^{2}$. Hence for given values of $a$ and $b$, we can get the values of $x, y, z$.

3 . Let $x^{2}-60=y^{2}$, then $(x-y)(x+y)=60$. Hence $x-y$ and $x+y$ must both be even. Therefore $x-y=2, x+y=30$ or $x-y=6, x+y=10$, thus $2 x=16$ or $x=8$.

4. From $x^{2}-5=y^{2}, x^{2}+5=z^{2}$, we get $10=z^{2}-y^{2}=(z-y)(z+y)$. But as one of $z-y$ and $z+y$ is even, the other is also even. Hence their product is a multiple of 4 , which is impossible. Therefore the required number $t$ does not exist.

5. We shall discuss the following three cases.

i) When $2 \nmid x, 2 \chi y$. Evidently $2 \chi z$. Taking the given equation for modulo 4 we get $3 \equiv 1(\bmod 4)$, which is impossible.

ii) When $2 \mid x$, taking modulo 4 , we get $y^{2}+z^{2} \equiv 0(\bmod 4)$. If one of $y$ and $z$ is odd, then $y^{2}+z^{2} \equiv 1$ or $\equiv 2(\bmod 4)$. This contradicts the above equation. Hence $x, y, z$ are all even. Putting $x=2 x_{1}, y=2 y_{1}, z=2 z_{1}$ and substituting in the given equation, we get $x_{1}^{2}+y_{1}^{2}+z_{1}^{2}=4 x_{1}^{2} y_{1}^{2}$. Similarly, taking modulo 4 , we get $x_{1} \equiv y_{1} \equiv z_{1} \equiv 0(\bmod 2)$. Putting $x=2 x_{2}, y=$ $2 y_{2}, z=2 z_{2}$, we also get $x_{2}^{2}+y_{2}^{2}+z_{2}^{2}=4 x_{2}^{2} y_{2}^{2}$. Taking modulo 4 again, we have $x_{2} \equiv y_{2} \equiv z_{2} \equiv 0(\bmod 2)$. Continuing this process, we obtain the same result. That is to say, if the given equation has solution, the solution can be divided by any powers of 2 . Hence $x=y=z=0$. 
iii) When $2 \mid y$, then in the same way, we have $x=y=z=0$.

6. The given equation can be written as $(y-x)(x+y-1)=18$. From $x \geq 1$ we get $2 x-1 \geq 1$. Thus $x+y-1=(y-x)+2 x-1>y-x$. Hence $y-x$ can take the values $1,2,3$, and $x+y-1$ must be $18,9,6$. That is,

$$
\left\{\begin{array} { l } 
{ y - x = 1 } \\
{ x + y - 1 = 1 8 }
\end{array} \quad \left\{\begin{array} { l } 
{ y - x = 2 } \\
{ x + y - 1 = 9 }
\end{array} \quad \left\{\begin{array}{l}
y-x=3 \\
x+y-1=9
\end{array} .\right.\right.\right.
$$

Solving, we get

$$
\left\{\begin{array} { l } 
{ x = 9 } \\
{ y = 1 0 }
\end{array} \quad \left\{\begin{array} { l } 
{ x = 4 } \\
{ y = 6 }
\end{array} \quad \left\{\begin{array}{l}
x=2 \\
y=5
\end{array} .\right.\right.\right.
$$

7. $z^{4}=\left(x^{4}-4 y^{4}\right)^{2}=x^{8}-8 x^{4} y^{4}+16 y^{8}=\left(x^{4}+4 y^{4}\right)^{2}-(2 x y)^{4}$, or $(2 x y)^{4}+z^{4}=\left(x^{4}+4 y^{2}\right)^{2}$.

8. As $x^{2}+1=3 y^{n}, x^{2}+1$ is a multiple of 3 . Let $x=3 k, 3 k+$ $1,3 k+2$. Substituting, we see that the corresponding $x^{2}+1$ all are not multiple of 3 . Hence the given equation has no integer solution.

9. Choosing a suitable positive integer $x$ such that $n-x^{2}=m$ is a positive odd number. Let $y=\frac{m+1}{2}$. Since $y^{2}-m=\left(\frac{m-1}{2}\right)^{2}=z^{2}$, then $n-x^{2}=y^{2}-z^{2}$.

10. Let $a, b, c$ be any system of solutions of the Shang Gao equation, then $\left(a c^{n-1}\right)^{2}+\left(b c^{n-1}\right)^{2}=\left(c^{2}\right)^{n}$.

11. When $x=1,2$ the given equation has no positive integer solution. Let $x>2$. Since $2 \nmid y, 3 \chi y$ and $y \neq 1$. Substituting $y=6 k t+1$ in the given equation we get

$$
3 \cdot 2^{x}+1=(6 k \pm 1)^{2}=36 k^{2} \pm 12 k+1,
$$

or

$$
2^{x-2}=3 k^{2} \pm k=k(3 k \pm 1)
$$


If $k=1$, we get two systems of solutions $(3,5)$ and $(4,7)$. If $k>1$, since the right-hand side of the above equation has an odd factor greater than 1 , and the left side has not, the given equation has no solution.

12. Since $\sum\left\{\left(2 n^{2}+2 n+i\right)^{2}-\left(2 n^{2}+n+i\right)^{2}\right\}=\sum n\left(4 n^{2}+3 n+2 i\right)=$ $\left(2 n^{2}+n\right)^{2}$, then the given equation exist. Taking $n=3$, we get $21^{2}+22^{2}+23^{2}+24^{2}=25^{2}+26^{2}+27^{2}$.

\section{Exercise 3.3}

1. $365=13^{2}+14^{2}, 1105=9^{2}+32^{2}, 1961=19^{2}+40^{2}$. But $5461=43 \cdot 127$ is not a sum of squares.

2. When $p=2$ or 5 , substituting in $2^{p}+3^{p}$ directly we easily see that it is not a square number. When $p \neq 2$ or $5, p=2 k+1$, then $2^{p}+3^{p}=(2+3)\left(2^{2 k}-2^{2 k-1} \cdot 3+\ldots+3^{2 k}\right)$ or $5 \mid\left(2^{p}+3^{p}\right)$. If $2^{p}+3^{p}$ is a square, then $2^{2 k}-2^{2 k-1} \cdot 3+\ldots+3^{2 k} \equiv 0(\bmod 5)$, and hence $2^{2 k}+2^{2 k}+\ldots+2^{2 k} \equiv 0(\bmod 5)$, or $p 2^{2 k} \equiv 0(\bmod 5)$. It is evidently impossible.

4. For $m=a^{2}-b^{2}=(a-b)(a+b), m=a b=\left(\frac{a+b}{2}\right)^{2}-\left(\frac{a-b}{2}\right)^{2}$.

5. $\left\{\frac{n(n+1)}{2}\right\}^{2}-\left\{\frac{(n-1) n}{2}\right\}^{2}=n^{3}$.

7. Let $a=(2 n)^{2}+(2 n)^{2}, b=8 n^{2}+1, c=(2 n-1)^{2}+(2 n+1)^{2}$. When $n=\frac{m^{2}+m}{2}$, then $a=2 m^{2}(m+1)^{2}, b=2 m^{2}(m+1)^{2}+$ $1, c=2 m^{2}(m+1)^{2}+2$. Hence $a, b, c$ are three consecutive numbers.

8. Let $x=a+3 d, y=a+4 d, z=a+5 d, w=a+6 d$. Substituting in the given equation we get $a\left(a^{2}+9 a d+21 d^{2}\right)=0$; hence $a=0$. Thus the required numbers are $x=3 d, y=4 d, z=5 d, w=$ $6 d$, where $d$ is any positive integer.

9. Since for modulo $p$ any two integers in $0,1^{2}, \ldots,\left(\frac{p-1}{2}\right)^{2}$ are incongruent and any two integers in $-1-0,-1-1^{2}, \ldots,-1-$ 
$\left(\frac{p-1}{2}\right)^{2}$ are also incongruent, these two sets are $2\left(\frac{p-1}{2}+1\right)=p+1$ in number. Hence there exists the congruent integers

$$
x^{2} \equiv-1-y^{2}(\bmod p)
$$

where $0 \leq x \leq \frac{p-1}{2}, 0 \leq y \leq \frac{p-1}{2}$.

\section{Exercise 4.1}

1. 1) $(256,337)=1$. It has a solution $x \equiv 81(\bmod 337)$.

2) $(1215,2755)=5,5 \mid 560$. It has 5 solutions

$$
x \equiv 200,751,1302,1853,2404(\bmod 2755) \text {. }
$$

3) $y=1+2 t, x \equiv-1-5 t+3 s(\bmod 18), t=0,1, \ldots, 8 ; s=$ $0,1, \ldots, 5$, which are $9 \cdot 6=54$ in number.

2. $x=4+49 t, y=-3+37 t$.

3. $x \equiv 93(\bmod 140)$.

4. 1) $x \equiv 8(\bmod 209)$.

2) $x \equiv-2(\bmod 77)$.

5. $x \equiv 6, y \equiv-1, z \equiv-8(\bmod 17)$.

6. $x=2531$.

7. $x \equiv 462 \cdot 3+385 \cdot 5+330 \cdot 4+210 \cdot 10 \equiv 2111(\bmod 2310)$.

8. If $\alpha \equiv a(\bmod m)$ or $m \mid(\alpha-a)$, then $p_{i}^{m_{i}} \mid(\alpha-a)$; hence $\alpha=$ $a\left(\bmod p^{m_{i}}\right)$. Conversely, if $\alpha \equiv a\left(\bmod p_{i}^{m_{i}}\right)$ or $p_{i}^{m_{i}} \mid(\alpha-a)$, then $\prod p_{i}^{m_{i}} \mid(\alpha-a)$; hence $\alpha \equiv a(\bmod m)$.

\section{Exercise 4.2}

1. $x \equiv 0,1, \pm 2(\bmod 5)$.

2. $x \equiv 1,-2, \pm 3(\bmod 7)$.

3. $x \equiv \pm 2, \pm 18(\bmod 41)$. 
4. Since $(p-1) !+1 \equiv 0(\bmod p), x \equiv-(p-x)(\bmod p)$, if $x$ takes the values $2,4, \ldots, p-1$, we have

$$
1^{2} \cdot 3^{2} \ldots(p-2)^{2} \equiv(-1)^{\frac{p-1}{2}}(p-1) ! \equiv(-1)^{\frac{p+1}{2}}(\bmod p) .
$$

If $x$ takes $1,3, \ldots, p-2$, we have

$$
2^{2} \cdot 4^{2} \cdots(p-1)^{2} \equiv(-1)^{\frac{p-1}{2}}(p-1) ! \equiv(-1)^{\frac{p+1}{2}}(\bmod p) .
$$

5. Since $n \mid(p-1)$, then $x^{p-1}-1=\left(x^{n}-1\right) g(x)$, where $g(x)$ is a polynomial in $x$ of the $(p-1-n)$ th degree. From Theorem 1, $g(x) \equiv 0(\bmod p)$ at most has only $p-1-n$ solutions. But $x^{p-1} \equiv 1(\bmod p)$ has $p-1$ solutions. Hence $x^{n}-1 \equiv 0(\bmod p)$ has at least $p-1-(p-1-n)=n$ solutions. Also from Theorem 1 , the number of solutions cannot be greater than this. Therefore it has exactly $n$ solutions.

6. If $f(x) \equiv 0(\bmod p)$ has $p$ different solutions, then from

$$
f(x)=\left(x^{p}-x\right) q((x)+r(x))
$$

we see that $r(x) \equiv 0(\bmod p)$ evidently has also $p$ different solutions. But the degree of $r(x)$ is less than $p$, hence the coefficients of $r(x)$ are all the multiples of $p$. Thus $f(x) \equiv\left(x^{p}-\right.$ $x) q(x)(\bmod p)$, that is, $x^{p}-x$ is a factor modulo $p$ of $f(x)$. The converse is also true.

\section{Exercise 4.3}

1. $x \equiv 22(\bmod 27)$

2. no solution

3. $x \equiv 1,7(\bmod 25)$

4. $x \equiv 2,5,11,17,20,26(\bmod 30)$

5. $x \equiv 1,4,11,14,(\bmod 15)$ 
6. $x \equiv 93(\bmod 125)$

7. $x \equiv 91(\bmod 120)$

8. For any odd prime $p$ the congruence $x^{2} \equiv 1(\bmod p)$ has two solutions $x=1, x=p-1$. If $p_{1}, \ldots, p_{k}$ are $k$ different odd primes, from Sun-Tsu theorem we see that the system of congruences $x \equiv a_{1}\left(\bmod p_{1}\right), \ldots, x \equiv a_{k}\left(\bmod p_{k}\right)$, where $a_{i}=1$, or $p^{i}-1, i=1, \ldots, k$, has $2^{k}$ different solutions modulo $m=$ $p_{1} \ldots p_{k}$. Clearly these solutions are also the $2^{k}$ different solutions of $x^{2} \equiv 1(\bmod m)$. Thus we can choose $k$ such that $2^{k}>n$. Hence we have $m>0$ such that $x^{2} \equiv 1(\bmod m)$ has more than $n$ different solutions.

\section{Exercise 5.2}

1. $\left(\frac{88}{109}\right)=1,\left(\frac{365}{1847}\right)=1,\left(\frac{-1457}{2389}\right)=-1$.

2. As $\left(\frac{17}{23}\right)=-1$, the given equation has no solution.

3. The quadratic residues of 23 are

$$
1,2,3,4,6,8,9,12,13,16,18 \text {. }
$$

The quadratic nonresidues are

$$
5,7,10,11,14,15,17,19,20,21,22 \text {. }
$$

4. Odd primes of which 7 is a quadratic residue are

$$
p=28 n \pm 1,28 n \pm 3,28 n \pm 9 .
$$

Odd primes of which 7 is a quadratic nonresidue are

$$
p=28 n \pm 5,28 n \pm 11,28 n \pm 13
$$

5. 1) $\left(\frac{p}{q}\right)=\left(\frac{q+4 a}{q}\right)=\left(\frac{4 a}{q}\right)=\left(\frac{a}{q}\right)$, 
2) $\left(\frac{q}{p}\right)=\left(\frac{p-4 a}{p}\right)=\left(\frac{-4 a}{p}\right)=\left(\frac{-1}{p}\right)\left(\frac{a}{p}\right)$.

Since $p \equiv q(\bmod 4)$, then $p \equiv 1(\bmod 4)$ or $p \equiv-1(\bmod 4)$. When $p \equiv 1(\bmod 4),\left(\frac{-1}{p}\right)=+1,\left(\frac{p}{q}\right)=\left(\frac{q}{p}\right) ;$ when $p \equiv$ $-1(\bmod 4),\left(\frac{-1}{p}\right)=-1,\left(\frac{p}{q}\right)=-\left(\frac{q}{p}\right)$. Hence we always have $\left(\frac{a}{p}\right)=\left(\frac{a}{q}\right)$.

6. If $\left(\frac{-1}{p}\right)=1$ then $p \equiv 1(\bmod 4)$ or $p=4 k+1$, that is, $p-1=$ $4 k,(p-1) !=(4 k) !$. Hence $1 \cdot 2 \cdots 2 k(2 k+1)(2 k+2) \cdots 4 k=$ $1 \cdot 2 \cdots 2 k(p-2 k)(p-2 k+1) \cdots(p-1) \equiv(1 \cdot 2 \cdots 2 k)^{2}(\bmod p)$.

7. As $x^{2}+3 \equiv 0(\bmod p),\left(\frac{-3}{p}\right)=\left(\frac{-1}{p}\right)\left(\frac{3}{p}\right)=\left(\frac{p}{3}\right), p^{\frac{3-1}{2}} \equiv 1$ $((\bmod 3)$, we have $p \equiv 1(\bmod 3)$ or $p=1+3 t$. As $p$ is odd, $t=2 k$ and hence $p=6 k+1$.

8. Since $x^{2} \equiv-y^{2}(\bmod p),\left(\frac{-y^{2}}{p}\right)=\left(\frac{-1}{p}\right)\left(\frac{y^{2}}{p}\right)=\left(\frac{-1}{p}\right)$, hence $p=$ $4 n+1$. Similarly from $x^{2} \equiv 2 y^{2}(\bmod p),\left(\frac{2 y^{2}}{p}\right)=\left(\frac{2}{p}\right)$, we get $p=8 n \pm 1$.

9. If $p_{1}, \ldots, p_{n}$ are $n$ prime numbers of the form $4 k+1$, then the odd prime factors of $\left(2 p_{1} \cdots p_{n}\right)^{2}+1$ are also of the form $4 k+1$. Similarly, the odd prime factors of $\left(2 q_{1} \cdots q_{m}\right)^{2}+3$ are also of the form $6 k+1$.

10. If $n$ is a prime number, from Theorem 3 , Sec. 1.4 we get $k \equiv$ $0(\bmod 2), n \equiv 1(\bmod 4)$. Thus $\left(\frac{3}{2^{k}+1}\right)=\left(\frac{2^{k}+1}{3}\right)=\left(\frac{2}{3}\right)=-1$. Hence the given congruence exists. Conversely, if the given condition exists, then $3^{n-1} \equiv 1(\bmod n)$. Let the order of 3 modulo $n$ be $\lambda$, then $\lambda \mid(n-1)=2^{k}$. If $\lambda<(n-1)$, putting $\lambda=2^{l}, l \leq k-1$, we get $3^{2^{k-1}} \equiv 1(\bmod n)$. This contradicts the hypothesis. Thus $\lambda=n-1$. Hence from Theorem 7, Sec. $2.2 n$ is prime.

\section{Exercise 5.4}

1. $x \equiv \pm 9(\bmod 19)$

2. $x \equiv \pm 11(\bmod 29)$

3. $x \equiv \pm 12(\bmod 71)$ 
4. $x \equiv \pm 21(\bmod 73)$

5. $x \equiv \pm 94(\bmod 353)$

6. $x \equiv \pm 11(\bmod 27)$

7. Multiplying the given equation by 8 we get $16 x^{2}+24 x+88 \equiv$ $0(\bmod 13)$, or $(4 x+3)^{2} \equiv-1(\bmod 13) ;$ that is $4 x+3 \equiv$ $1(\bmod 13)$ and $4 x+3 \equiv-1(\bmod 13)$. Thus the required solutions are $x \equiv 6(\bmod 13)$ and $x \equiv-1(\bmod 13)$.

\section{Exercise 5.5}

1. $x \equiv \pm 19, \pm 19+32(\bmod 64)$

2. $x \equiv \pm 108(\bmod 343)$

3. $x \equiv \pm 41, \pm 41+128(\bmod 256)$

4. $x \equiv \pm 113, \pm 141(\bmod 508)$

5. Evidently the four solutions $x \equiv \pm \alpha, \pm \alpha+2^{k-1}\left(\bmod 2^{k}\right)$ of (3) are also the solutions of (4). For modulo $2^{k}$, these four solutions are different; but for modulo $2^{k-1}$, they are essentially two different solutions. That is to say, the four solutions of (3) are actually only two solutions of (4). For instance the solutions of $x^{2} \equiv 1\left(\bmod 2^{3}\right)$ are $\pm 1, \pm 1+4$ or $1,3,5,9$, while the solutions of $x^{2} \equiv 1\left(\bmod 2^{4}\right)$ are $\pm 1, \pm 1+8$, or $1,7,9,15$. Note that here $1 \equiv 9\left(\bmod 2^{3}\right), 7 \equiv 15\left(\bmod 2^{3}\right)$.

\section{Exercise 6.1}

1. 1) $a^{p} \equiv 1(\bmod q), a^{\lambda} \equiv 1(\bmod q)$. When $\lambda \mid p$, then $\lambda=1$ or $\lambda=p$. When $\lambda=1$, then $a-1 \equiv 0(\bmod q)$ or $q \mid(a-1)$. When $\lambda=p$, then $\lambda \mid \phi(g)=q-1$, where $q=p t+1$. But as $q$ is odd, $q=2 p m+1$.

2) $a^{2} \equiv-1(\bmod q)$ or $(-a)^{p} \equiv 1(\bmod q)$, hence $q \mid(-a-1)$. Thus $q \mid(a+1)$ or $q=2 p m+1$. 
2. If $g$ is a primitive root of $p$, then $1, g, g^{2}, \ldots, g^{p-2}$ is the reduced residue system of $p$. Since $1,2, \ldots, p-1$ is also the reduced residue system of $p, 1^{n}+2^{n}+\ldots+(p-1)^{n} \equiv 1^{n}+g^{n}+$ $g^{2 n}+\ldots+g^{(p-1) n}=\frac{g^{(p-1) n}-1}{g^{n}-1}(\bmod p)$. But $g^{p-1} \equiv 1$ modulo $p$ and $n$ is not a multiple of $p-1$, thus $g^{n}-1 \not \equiv 0(\bmod p)$. Hence the given congruence exists.

3. Since $\alpha^{\lambda_{1}} \equiv 1\left(\bmod p_{1}\right), \alpha^{\lambda_{2}} \equiv 1\left(\bmod p_{2}\right)$, if $m=\left[\lambda_{1}, \lambda_{2}\right]$ then $\left(\alpha^{\lambda_{1}}\right)^{\frac{m}{\lambda_{1}}} \equiv 1\left(\bmod p_{1}\right),\left(\alpha^{\lambda_{2}}\right)^{\frac{m}{\lambda_{2}}} \equiv 1\left(\bmod p_{2}\right) ;$ hence $\alpha^{m}$ $\equiv 1\left(\bmod p_{1} p_{2}\right)$. Let the order of $\alpha$ modulo $p_{1} p_{2}$ be $\lambda$, then $\lambda \mid m$. Since $\alpha^{\lambda} \equiv 1\left(\bmod p_{1} p_{2}\right)$,

$$
\alpha^{\lambda} \equiv 1\left(\bmod p_{i}\right), \quad i=1,2,
$$

hence $\lambda_{1} \mid \lambda$. Thus $m \mid \lambda$, and therefore $\lambda=m$.

4. Let $a$ be the quadratic nonresidue of $p$. Then $a^{\frac{p-1}{2}}=a^{2^{m-1}} \not \equiv$ $1(\bmod p)$. But as $a^{p-1}=a^{2^{m}} \equiv 1(\bmod p)$, the order of $a$ is $2^{m}=p-1$. Thus $a$ is a primitive root of $p$.

5. Since $t_{1}, \ldots, t_{k}$ is the reduced residue system of $p-1$, there exists a divisor $d$ of $p-1$ such that $t_{i} \not \equiv t_{j}(\bmod d)$, where $t_{i}, t_{j}$ are any two integers in $t_{1}, \ldots, t_{k}$. Evidently $h=g^{\frac{p-1}{d}}$, where $g$ is a primitive root of $p$, is the required integer.

\section{Exercise 6.2}

1. The primitive roots of 23 are the following 10 integers:

$$
5,7,10,11,14,15,17,19,20,21 \text {. }
$$

The primitive roots of 54 are

$$
5,11,23,29,41,47 \text {. }
$$

There are 6 in number. Moreover, 5 is also a primitive root of 23 , it is also the primitive root of $529=23^{2}$ and $1058=2 \cdot 23^{2}$. 
2. If the order of $g$ is $\lambda$ and $\lambda<p-1$, then $\lambda \mid(p-1)$. Let $p-1=\lambda d$. When $2 \mid d$ or $d=2 d_{1}$, then $\lambda d_{1}=\frac{p-1}{2}, g^{\lambda d_{1}}=$ $\left(g^{\lambda}\right)^{d_{1}} \equiv 1(\bmod p)$, that is, $g^{\frac{p-1}{2}} \equiv 1(\bmod p)$, which is impossible. When $q_{i} \mid d$ or $d=q_{i} d_{i}$, then $\lambda d_{i}=\frac{p-1}{q_{i}}$. Hence $g^{\lambda d_{i}} \equiv\left(g^{\lambda}\right)^{d_{i}} \equiv 1(\bmod p)$, that is, $g^{\frac{p-1}{q_{i}}} \equiv 1(\bmod p)$, which is also impossible. Thus $\lambda=p-1$. Therefore $g$ is a primitive root of $p$.

3. Let $a$ be the quadratic nonresidue of $p$ and the order of $a$ modulo $p$ be $\lambda$. Then $\lambda \mid \phi(p)=2^{k}$. If $\lambda<2^{k}$, putting $\lambda=2^{l}, l<k$, we have $a^{2^{l}} \equiv 1(\bmod p)$ and hence $a^{\frac{p-1}{2}} \equiv 1(\bmod p)$. This contradicts the hypothesis. Hence $\lambda=2^{k}=p-1$.

4. Since the order of 2 modulo 41 is 20 , the following integers

$$
\begin{aligned}
& 2,4,8,16,32,23,5,10,20,40 \\
& 39,37,33,25,9,18,36,31,21,16
\end{aligned}
$$

are all not primitive roots of 41 . Also since the order of 3 modulo 41 is 8 , the integers

$$
3,9,27,40,38,32,14,1
$$

are all not primitive roots of 41 . Thus among the reduced residue system of 41 , we have besides these, $\phi(40)=16$ integers

$$
\begin{aligned}
& 6,7,11,12,13,15,17,19, \\
& 22,24,26,28,29,30,34,35
\end{aligned}
$$

which are all primitive roots of 41 .

\section{Exercise 6.3}

1. 2 is obtained immediately by Theorem 2 . 


\section{Exercise 6.4}

1. The quadratic residues are $1,4,5,6,7,9,11,16,17$, the cubic residues are $1,7,8,11,12,18$.
2. 1) $x \equiv 33(\bmod 67)$
2) $x \equiv 59,11,39(\bmod 109)$
3) no solution

3. Since $a \equiv-b(\bmod p)$, ind $a=$ ind $(-1)+$ ind $d$, and hence ind $a-$ ind $b \equiv \frac{p-1}{2}(p-1)$.

4. Let $h^{l_{1}} \equiv a(\bmod p), g^{l_{2}} \equiv a(\bmod p), h^{l_{3}} \equiv g(\bmod p)$. Then $h^{l_{2} l_{3}} \equiv g^{l_{2}} \equiv h^{l_{1}}(\bmod p)$. Hence $l_{1} \equiv l_{2} l_{3}(\bmod (p-1))$. 
This page is intentionally left blank 


\section{INDEX}

algebraic integer, 213

algebraic integral ring, 214

algebraic numbers, 213

algebraic number field, 214

algebraic number theory, 214

analytic number theory, 208

base, 5

Chebyshev theorem, 209

Chen's theorem, 211

common divisor, 9

common multiple, 16

complete residue system, 58

composite number, 21

concentration, 172

congruence, 52

Diophantine equation, 77

Dirichlet's theorem, 210

divisor, 1

elementary number theory, 207

Euclidean algorithm, 10

Euler's constant, 216

'vler's criterion, 143

er function, 61

? Goldbach's conjecture, 210

'ven number, 2

Fermat number, 39

Termat's greatest theorem, 93

wat's last theorem, 93

nat's little theorem, 63
Fermat's theorem, 63

function of number theory, 72

fundamental theorem of arithmetic, 27

Gauss function, 42

Gauss integral point problem, 215

Gauss lemma, 146

geometric number theory, 217

Goldbach's conjecture, 210

greatest common divisor, 9

ideal number, 214

indeterminal equation, 77

index, 198

integer point, 13

Jacobi symbol, 159

lattice point, 215

law of quadratic reciprocity, 151

least common multiple, 16

Legendre symbol, 144

Mersenne number, 37

Minkowski function, 216

Möbius function, 48

multiple, 1

multiple solution, 125

multiplicative function, 72

number-theoretic function, 72 
odd Goldbach's conjecture, 210 odd number, 2

order, 64

pairwise relatively prime, 14 perfect number, 35

prime number, 21

prime number theorem, 208

primitive root, 182

proper divisor, 2

Pythagorean equation, 87

quadratic nonresidue, 139

quadratic residue, 139

recurrent integer, 35

reduced residue system, 58

relative prime, 12
Shang-gao indeterminate equation, 85

Shang-gao number, 87

sieve, 25

simple solution, 125

solution, 109

square number, 4

Sun-Tsu theorem, 116

three primes theorem, 211

triangular number, 105

transcendental number, 214

transcendental perfect number,

42

twin primes, 211

twin primes conjecture, 211

Waring's conjecture, 212

Wilson's theorem, 128 